\title{
Study on Feature Extraction Method of Han Dynasty Stone Carving Images
}

\author{
WANG Juan \\ Software College \\ Nanyang Institute of Technology \\ Nanyang, China \\ juanwj129@gmail.com
}

\author{
TANG Man \\ Software College \\ Nanyang Institute of Technology \\ Nanyang, China \\ tangman131421@163.com
}

\begin{abstract}
Han Dynasty stone carving is Chinese nation's outstanding cultural heritage, how to protect and promote it is an historical responsibility. In recent years, there has been a lot of research on the Han Dynasty stone carving, and has achieved certain results. But the digital research on it is still very rare. This is mainly because the own characteristics of Han Dynasty stone carving images make feature extraction become difficult. Feature extraction is the key point of Han Dynasty stone carving's digital research, which directly affects the accuracy of image retrieval and the efficiency of image matching algorithm. This paper based on the analysis of the characteristics of Han Dynasty stone carving images and the existing image feature extraction methods, puts forward a scheme for feature extraction of Han Dynasty stone carving images which adopts multi- feature fusion.
\end{abstract}

Keywords- Han Dynasty stone carving images; feature extraction; image retrieval; multi-feature fusion

\section{INTRODUCTION}

Han Dynasty stone carving is the treasure of Chinese culture, and it is a giant rock epic which depicts the Han Dynasty life, politics, economy and other aspects through vivid pictures. Along with more and more Han Dynasty stone carvings to be found and excavated, image recognition and image retrieval workload is increasing during the Han Dynasty stone carving image research. At present, there are many website about the Han Dynasty stone carving such as the website [1], in which the Han stone carving images were classified and display, but the digital research on it is still very rare. There is no complete digital resource library for the Han Dynasty stone carvings matching and retrieval, which can deal with stone carving image preprocessing, feature extraction and image retrieval. Research on the Han Dynasty stone carving image retrieval system, and to establish a digital resource library and a feature database, can not only realize the Han Dynasty stone carving's digital storage, but also realize the image retrieval, which is contribute to the protection and the study of Han Dynasty stone carving.

Han Dynasty stone carving image retrieval system is a new application of image processing technology in the field of pattern recognition. Firstly, collect Han Dynasty stone carving images and related information. Then, take image pretreatment. After that, select and research appropriate segmentation strategy and algorithms in connection with the complexity of the background and target, at the same time, study the affect of image scaling and rotating to image feature extraction. Then, study the characteristics of Han Dynasty stone carving images, analyze the effective features and propose effective schemes for feature extraction, and establish a Han Dynasty stone carving images digital feature library and retrieval images based on it. Last, establish a retrieval system. Among them, the appropriate and effective feature extraction method is the key point, which directly impact on the determination of image detection algorithm, the accuracy of image matching and the efficiency of matching algorithms. And it has a decisive role on the formation of feature database about Han Dynasty stone carving images and effective image retrieval.

\section{DESIGN OF HAN DYNASTY STONE CARVING IMAGE RETRIEVAL SYSTEM}

Han Dynasty stone carving image retrieval system is a specific application of pattern recognition technology. Pattern recognition technology ${ }^{[2][3]}$ continues to play an important supporting role in the development of image understanding and robot vision. Image recognition technology based on feature matching is a hot research at home and abroad, and also the key technology of future information superhighway, digital libraries and other major projects.

In view of the Han Dynasty stone carving, the method of on-the-spot investigation, photographed and resource search will to be used, first of all, establish an image library of Han Dynasty stone carving images, after that, study feature extraction method and carry out theoretical analysis and experimental research based on related image processing and pattern recognition theory and algorithm, study of Han Dynasty stone carving image, in the above foundation, study image detecting and matching methods, and eventual establish a Han Dynasty stone carving image retrieval system. The overall system flow was shown in figure 1.

- $\quad$ First of all, collect related data of Han Dynasty stone carving images, preprocessing them, and establish an image library of Han Dynasty stone carving images.

- Then, carry out feature extraction on Han Dynasty stone carving images. According to the characteristics of Han Dynasty stone carving images, analyze the effective features and propose effective schemes for 
feature extraction, establish the Han Dynasty stone carving image digital feature library.

- Preprocess the query image and extract features according to the scheme for feature extraction of Han Dynasty stone carving images.

- Match the query image in the digital resource library, take the match precision and speed as the algorithm measure, and study the most similar image's retrieval in case of image deformation and defect.

- Finally, select the appropriate image preprocessing algorithm, image feature extraction method and image matching algorithm for system integration, and establish a Han Dynasty stone carving image retrieval system.

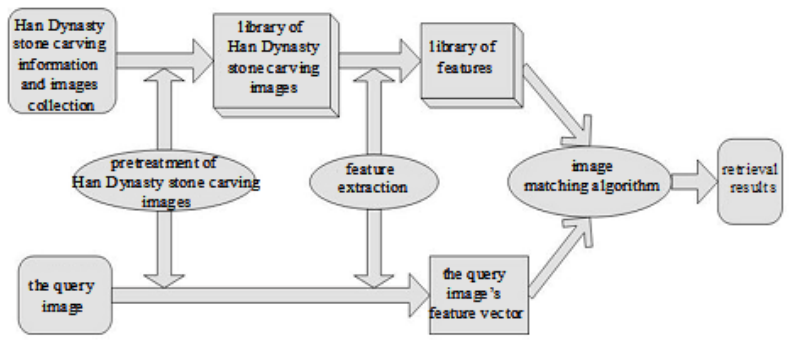

Figure 1. Han Dynasty stone carving image retrieval system

The image matching method has two main categories: gray based matching method and feature based matching method. Gray scale matching method directly uses gray-scale for image matching; the algorithm is simple and has high matching accuracy, but it has a large amount of computation. Image feature based matching methods have to extract image features at first; the calculation is relatively small, with wide application field, which is a hot research topic at present. The image matching theory and technology are close related to practical application, the general theory and technology need improvement according to practical application, and then form applicable specific theory and technology to a particular field.

Feature extraction method is the foundation of Han Dynasty stone carving image retrieval system, there is still no systematic research and application for feature extraction point at Han Dynasty stone carving images. Therefore, study the scheme for Han Dynasty stone carving images has a certain practical significance and application value.

\section{ANALYSIS OF THE CHARACTERISTICS OF HAN DYNASTY STONE CARVING IMAGES}

Han Dynasty stone carving images are often derived from shoot or rubbing, it is not conducive to images' digital processing due to light, angle and other factors. At the same time, the own characteristics increase the difficulty for feature extraction. Before feature extraction of Han Dynasty stone carving images, they need to pretreatment, make the image into required size, and take gray processing. But there are still two difficulties for Han Dynasty stone carving images' feature extraction:
- Han Dynasty stone carving images are mostly as a strip shape, the pictures are mostly local image and have different size, and the same image has multiple targets, which is not conducive to the feature extraction.

- The background of Han Dynasty stone carving image is complicated, the background region and the target region often have high similarity, which also increases the difficulty for feature extraction of Han Dynasty stone carving images.

It needs to choose suitable image segmentation algorithm because of the multiple targets and large size of Han Dynasty stone carving images. Image segmentation is the basic work of target detection and image recognition. There has raised thousands of segmentation algorithms in many literature, but in practical application, there is still not a generally applicable method because of the complexity of background and objectives. Specific segmentation method can only be applied in specific applications. So in the concrete issue of Han Dynasty stone carving images, to select and study appropriate segmentation strategy and algorithm is a very important basic work.

Due to the complicated background of Han Dynasty stone carving images, background region and target region have high similarity, when to extract feature, it is need to have a reasonable description of target characteristics and background characteristics first of all. Therefore, we consider the use of top-down knowledge driven strategy method for target recognition. According to Han Dynasty stone carving images' characteristics, establish a correct knowledge expression model, it is advantageous to rapid detection and targets identification for wide range image including complex background. So far, the simple and effective method is to select an appropriate threshold, binary-processing on the image, as the background area (pixel value is 0) removed, retained the target area (pixel value is 1$)$.

\section{STUDY ON FEATURE EXTRACTION METHOD OF HAN DYNASTY STONE CARVING IMAGES}

The research work of image feature extraction has been reported so far, and there are a lot of feature extraction methods $^{[4][5][6]}$, such as color or gray-scale statistical feature extraction, texture or edge feature extraction, image algebra feature extraction, image transform coefficients feature extraction, etc. Each method has its own advantages and disadvantages. The key issues facing feature extraction of stone carving images are what the most important feature of Han stone carving images is and how to choose the appropriate feature extraction method.

\section{A. feature analysis of stone carving images}

Feature selection is the foundation and key of pattern recognition. Feature selection will affect the classifier's quality directly. Therefore, each step is very important including original feature, feature selection and feature extraction. Based on the characteristics of Han stone carving images and combined with the existing methods for image feature extraction, this paper selects the appropriate and effective 
features and explores suitable feature extraction method for Han stone carving images.

The processed image based on feature matching generally contains color features, texture features, shape features and spatial features etc. Therefore, the following analyzes several features of Han stone carvings image.

1) Color feature and spatial feature: Han stone carvings image has no obvious color features. With the features of the spatial relationships between the objects image retrieval involves early image segmentation and object region to obtain. And the object region in Han stone image is complex and difficult to obtain.

2) Texture features: Texture refers to a visual pattern that has properties of homogeneity that do not result from the presence of only a single color or intensity ${ }^{[5]}$. Image retrieval based on texture feature is on the basis of the color, considered the relationship between the adjacent pixels, and has regularity, roughness, direction, etc. to measure the image linear feature. Han stone carvings image has no obvious color features and the background region and object region often have high similarity. In pursuit of the optimal solution at the same time cost is the smallest to extract image features. So the image should be processed into binary image. Binary image mainly reflects structural features in image and ignores texture, luminance, contrast, etc. Texture feature extraction has no advantage in the binary image. Therefore, texture feature is not fit for Han stone carving image retrieval.

3) Shape feature: In the Shape-based Image Retrieval image is divided into closed region. Image details such as background elements are shielded that is closer to the human cognitive faculty. Shape analysis of object can base on both the region itself and the boundary of the region. The shape features of the region itself include geometric features ${ }^{[6]}$, such as area, perimeter, roundness, convexity and concavity, the Moment Feature such as the centroid, central moments, $\mathrm{Hu}$ moments, Zernike moments, wavelet moment, flat, etc., as well as the Euclidean distance; The shape features of the region boundary are usually described by Fourier operator or chain code to represent. The object region of most Han stone carving image is composed of relatively clear boundary geometry. So Han stone carving image is seen as some regions which have a significant shape features.

\section{B. Feature selection of stone carving images}

Image feature extraction technology appears the development trend of multi-feature fusion. In addition to the use of the original image gray feature, also can use image visual features, statistical characteristic and transform coefficient features, through multi-feature fusion, the image pixels can be fully described, which can get a better extraction results. Aiming at the shape of Han stone carving images the following analyzes contour feature, Moment Invariants and Scale Invariant Feature Transform, and chooses appropriate feature extraction methods for Han stone carving images.

\section{1) Edge contour feature}

The edge is one of the most basic features of an image. Edge refers to those pixels set which the surrounding pixel gray value has a step change or slope change ${ }^{[6]}$. Edge has two characteristics of direction and amplitude. Along the edge gray values change gently, while perpendicular to the edge changes sharp and may be a step or slope change. In order to obtain a clear contour of object in binary image, erosion operation in binary mathematical morphology can be used in contour extraction. Erosion operation methods in binary mathematical morphology: for arbitrary pixel point of image, if its own pixel value and its peripheral four pixel value are 1 , then the pixel value is 0 .

Fourier descriptor is an important method used in shape analysis and recognition. The Fourier descriptors algorithm is to compute Fourier transform for mark function of boundary (such as the boundaries of complex coordinates, centroid distance and boundary curvature), to obtain Fourier coefficients as a descriptor. Contour feature of object region can describe by Fourier transform. Through Fourier transform the contour feature quantity is converted to the frequency domain, and then using low-frequency coefficients to approximate representation of contour. The low-frequency coefficients express the main body of the contour feature quantity, so it is possible to express a contour with fewer coefficients.

Object region feature extraction of Han stone carving image can be based on the edge contour detection, and then use the Fourier transform to obtain Fourier descriptors as the basis of Han stone carving image matching.

\section{2) Moment Invariants}

The image moments feature is a method to describe the statistical characteristics of gray scale. Moment feature is a statistical average description, and can describe object feature from global viewpoint. In 1962, Hu Minggui proposed a set consisting of seven invariant moments ${ }^{[7,8]}$. Hu moments after correction became important parameter to describe the image. $\mathrm{Hu}$ moments have the advantages of invariance for image rotation, scale and translation. So it can describe image region features and has been widely used in the field of image recognition. At the same time, moment invariants also evolved into a variety of forms, such as Zernike moments, wavelet moment and boundary moment. The boundary moment is based on the moments calculated by the image contour.

Moment function is less sensitive to noise. Whether the object is closed, it can better identify the object. The background of Han stone carving images is complicated and background region and object region have high similarity. So feature extraction using Hu moments or boundary moment can greatly reduce the influence of external factors on the image matching.

The principle of Han stone carving image feature extraction is to find the most prominent features and to get the optimal solution using minimum cost. A lot of research was done. And other methods have limitations for Han stone carving images such as scale invariant feature transform (SIFT) ${ }^{[9]}$. The SIFT algorithm runs slowly and has mismatching in the region with similar gray change. Although the algorithm is a very good 
application in many areas, but it is not fit for the Han stone carving images.

\section{Multi-feature fusion}

Because of diversity and uncertainty of objects imaging, a single feature extraction method is difficult to obtain satisfactory results for images that contain complex object. Multi-feature fusion makes the extracted features associated together; eigenvectors transform into meaningful combinations, and then analyze and process the associated features comprehensively ${ }^{[10]}$.

We studied feature extraction methods aiming at the unique features of Han Dynasty stone carving images. We discussed texture feature and shape feature, and analyzed several features including edge contour feature, region Moment Invariants, boundary moment and SIFT. And there are the following conclusions: based on the calculation of the boundary moments in contour feature extraction and combined with $\mathrm{Hu}$ moments of Han stone carving images, multi-feature fusion can restrict Han stone carving images' matching process; and it will improve the accuracy of image retrieval. The multi-feature fusion process is shown in Figure 2:

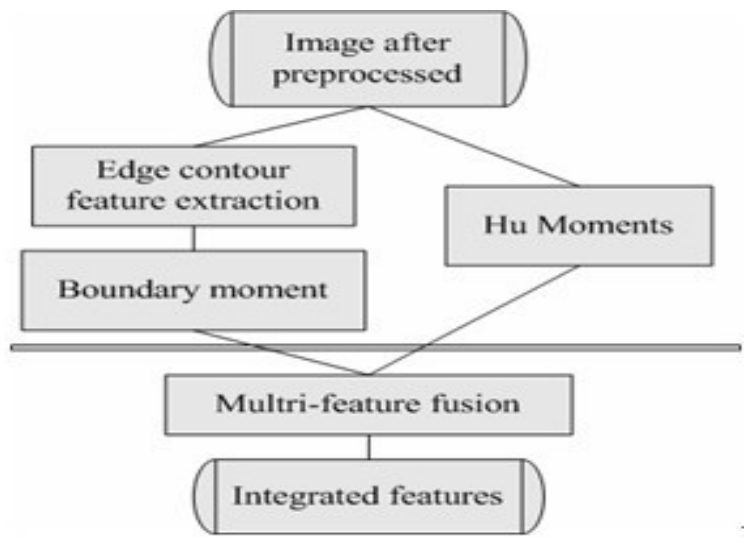

Figure 2. multi-feature fusion process

\section{CONCLUSION}

The establishment of Han stone carving image retrieval system needs several steps including image collection, preprocessing, feature extraction and image matching. Feature selection and extraction directly affect the image matching accuracy and efficiency of matching algorithm. The principle of Han Stone Carvings image feature extraction is to find the most prominent features and to get the optimal solution using minimum cost.

According to the characteristics of Han stone carving images, this paper analyzes several features including edge contour feature, region Moment Invariants, boundary moment and SIFT. Based on the boundary moments in Contour feature extraction and combined with $\mathrm{Hu}$ moments of Han Stone Carvings image, multiple feature fusion can be used in Han Stone Carvings image matching process.

The scheme helps to build the Han stone image digital feature library. It have the practical significance in the construction of the Han Dynasty stone carving's digital resources, the protection and development of stone carving and the Han Dynasty culture. At the same time, it also enriches the applications of pattern recognition and feature extraction techniques.

\section{REFERENCES}

[1] Han Dynasty stone carving database in China. http: //202.195.72.39 /tpi_14/sysasp/include/index.asp.

[2] A. Rosenfeld and J. Weszka, "Picture recognition" in digital pattern recognition, K. Fu (Ed.), Springer-Verlag, 1980, pp.135-166.

[3] Yong Rui and Thomas S. Huang, "Image retrieval: current techniques, promising directions, and open issues," Journal of visual communication and image representation, 1999, vol. 10, pp. 39-62.

[4] Rochan Shrestha, Image similarity matching based on colour and texture, Changsha, China: Hunan University, 2005.

[5] G. N. Srinivasan and Shobha G., "Statistical texture analysis," Proceedings of world academy of science, engineering and technology, vol. 36, pp.1264-1269, December 2008.

[6] Sven Loncaric, “A survey of shape analysis techniques,” Pattern recognition, vol. 31, pp. 983-1001, August 1998.

[7] HU M. K. "Visual pattern recognition by moment invariants," IRE transactions on information theory, 1962, vol. 8, pp. 179-187.

[8] Jan Flusser, "Moment Invariants in Image Analysis," World academy of science, engineering and technology, 2005, pp. 376-381.

[9] D G Lowe. "Distinctive image features from scale-invariant key-points," International journal of computer vision, 2004, vol. 60, pp. 91-110.

[10] Reinhold Huber-Mörk, Sebastian Zambanini, Maia Zaharieva and Martin Kampel, "Identification of ancient coins based on fusion of shape and local features,” Machine vision and applications, 2011, vol. 22, pp.983-994. 